\title{
A network approach to the analysis of psychosocial risk factors and their association with health
}

\section{Elovainio, Marko}

2020-09

Elovainio , M , Hakulinen , C, Pulkki-Raback, L, Juonala , M \& Raitakari , O T 2020 , ' A network approach to the analysis of psychosocial risk factors and their association with health ' , Journal of Health Psychology , vol. 25 , no. 10-11 , pp. 1587-1600 . https://doi.org/10.1177/1359105318765

http://hdl.handle.net/10138/322236

https://doi.org/10.1177/1359105318765624

cc_by_nc_nd

acceptedVersion

Downloaded from Helda, University of Helsinki institutional repository.

This is an electronic reprint of the original article.

This reprint may differ from the original in pagination and typographic detail.

Please cite the original version. 
Running title: Network structure of psychosocial risks

A network approach to the analysis of psychosocial risk factors and their association with health

\section{Marko Elovainio, $\mathrm{PhD}$}

Department of Psychology and Logopedics, Faculty of Medicine, University of Helsinki, Finland and the National Institute for Health and Welfare, Finland

\section{Christian Hakulinen, $\mathrm{PhD}$}

Department of Psychology and Logopedics, Faculty of Medicine, University of Helsinki, Finland and the National Institute for Health and Welfare, Finland

Laura Pulkki-Råback, $\mathrm{PhD}$

Helsinki Collegium for Advanced Studies, University of Helsinki, Finland;

Markus Juonala, MD, $\mathrm{PhD}$

Research Centre of Applied and Preventive Cardiovascular Medicine, University of Turku and Department of Clinical Physiology and Nuclear Medicine, Turku University Hospital, Turku, Finland.

Olli T. Raitakari, MD, PhD

Research Centre of Applied and Preventive Cardiovascular Medicine, University of Turku and Department of Clinical Physiology and Nuclear Medicine, Turku University Hospital, Turku, Finland.

Correspondence: Marko Elovainio, University of Helsinki, P.O. Box 9, 00014, Helsinki, Finland, Phone: +358 50 3020621, email: marko.elovainio@ helsinki.fi

Word count for abstract / main text: 4800

Number of tables: 2

Number of figures: 3

Competing interests: None. The funders were not involved in the design and conducting of the study, in the collection, management, analysis and interpretation of the data, nor in the preparation, review or approval of the manuscript. 


\begin{abstract}
We modelled early psychosocial risks as a network of interconnected variables, to study their associations with later depressive symptoms and cardiometabolic outcomes. The participants were a nationally representative sample of 2,580 men and women aged 3-18 years in 1980. Their parents reported the psychosocial risks in 1980, including: 1) child-specific life events, 2) parental health behaviour, 3) parental socioeconomic status and 4) parental psychological problems. Adulthood depressive symptoms and cardiometabolic outcomes were measured in 2007-2012. The most central risks (most number of connections to other risks) were socioeconomic risks that also predicted health outcomes more consistently than others.
\end{abstract}

Keywords: Network, Causality, Health risks, Epidemiology, Psychosocial 
It is well known that early exposure to adverse environments in childhood (such as poor housing, economic pressure) or traumatic events are associated with later health problems, including depressive symptoms, sickness absences, hospitalization, number of chronic illnesses, over weight as well as lipid and carbohydrate metabolism-related risk factors (Elovainio, Pulkki-Raback, et al., 2015; Evans, Dongping, \& Whipple, 2013; Kawachi, Adler, \& Dow, 2010; Repetti, Taylor, \& Seeman, 2002; Slopen, Goodman, Koenen, \& Kubzansky, 2013; Taylor, Repetti, \& Seeman, 1997). Furthermore, according to recently provided evidence, accumulated multiple exposures are likely to have a more profound influence on child development than single exposures (Puolakka et al., 2016; Juonala et al., 2016; Hakulinen et al., 2016; Elovainio et al., 2016; Elovainio et al., 2015b), thus providing a more comprehensive picture of the early factors that lead to future health problems (PulkkiRaback et al., 2015b).

Although the significance of risk accumulation is well-established, less is known about the relationships and inter-connections between individual risks that are often treated as interchangeable in cumulative models. These individual risks are traditionally conceived of as independent (Evans et al., 2013) and additive (DiPrete and Eirich, 2006), but it is also reasonable to assume that they are connected, and that some are more tightly and densely connected than others. In the following we propose a new way of thinking about the widely studied psychosocial risks and their development as webs of interlinked risks in a mutually related network (Cramer et al., 2010; Bringmann et al., 2013). This new approach - network analysis - has recently been applied to the study of mental-health problems and symptoms, and this line of research is rapidly expanding (Bringmann et al., 2013; Borsboom and Cramer, 2013; Kossakowski et al., 2016). The reason for the rapid growth is twofold, relating to: (A) 
the development of statistical tools for analysing networks (Kruis and Maris, 2016; van Borkulo et al., 2014; Epskamp et al., 2012), and (B) the construction of new theoretical frameworks that enhance understanding of mental-health problems (Boschloo et al., 2015; McNally et al., 2017), personality traits (Costantini et al., 2014), health measures (Kossakowski et al., 2016) and attitudes (Dalege et al., 2016) as network structures. The network framework as a concept focuses on individual variables that may have unique sets of associations with other variables. These associations could be visualized and illustrated as a network in which individual variables are nodes and the causal interactions between them are the connecting mechanisms.

This approach is new in psychiatry not only because of the statistical tools applied (van Borkulo et al., 2015; Boschloo et al., 2016; Frewen et al., 2013), but also because it represents a fundamental shift in thinking about mental disorders. The classic view is that mental-health problems and their causes arise from common disorders (Common Cause -hypothesis) (Kendler, 2012; Borsboom and Cramer, 2013; Fried et al., 2017). According to the recently developed Network Spread hypothesis, however, the main mechanisms underlying mental disorders work through the spreading of symptoms in causal networks (Borsboom and Cramer, 2013). Hence, the symptoms of depression may activate (or cause) each other in a certain pattern: for instance, insomnia may cause fatigue that in turn causes concentration problems that may lead to negative perceptions of one's own performance, and so forth. Moreover, some of these patterns are more central than others in the network of interrelated multiple risks, potentially leading to other negative outcomes, such psychoses or major depressive episodes (van Borkulo, Boschloo, Borsboom, \& al., 2015; van Borkulo et al., 2015). 
The most recent research based on the Network Spread approach focuses on mental disorders, although it could profitably be applied to several other phenomena. The area of psychosocial risk factors has not, to our knowledge, been explored from this perspective. As an approach, it could offer new explanations related to two unanswered questions in research on the association between psychosocial risk and health. First, how are the socioeconomic and other psychosocial risks interconnected and second, does the position of an individual risk in the risk network (its relations to other risks) associate with mental and physiological health outcomes (Stringhini et al., 2017)? We posit that the psychosocial risks that are more central than others in the network, and thus are theoretically more efficient in affecting (or associating with) the other risks, may be the ones that also have stronger associations with health outcomes. Thus, the network approach may help to inform which factors are the most interesting of the psychosocial risks for the prediction of health outcomes. Based on previous literature we expected that socioeconomic risks are particularly central in the network (i.e. have many and/or strong associations) and thus activate multiple other risks (Marmot et al., 1991; Marmot and A, 1996; Singh-Manoux et al., 2003; Elovainio et al., 2011)

The present study applies the network approach in exploring the interconnections between early psychosocial risks and their associations with later depressive symptoms and cardiometabolic factors (body mass index (BMI), intima media thickness (IMT) and type 2 diabetes) in a prospective Young Finns study with up to 32 years of follow-up. These outcomes were chosen because they are highly prevalent in the population and impose a significant burden on health-care and well-being, and they all have also been associated with early psychosocial risks (DALYs and Collaborators, 2017; Baltrus et al., 2007; Elovainio et al., 2015b; Elovainio et al., 
2016; Elovainio et al., 2012; Hakulinen et al., 2016; Juonala et al., 2016; Kivimaki et al., 2006; Okasha et al., 2003; Tamayo et al., 2010). We thus (A) explored the network structure of early psychosocial risks (socioeconomic, emotional, stressful events, parental health behaviours) that previous studies have shown to be associated with later health-related outcomes including physical health risks and depressive symptoms (Elovainio, Pulkki-Raback, et al., 2015; Elovainio et al., 2016; Hakulinen et al., 2016; Juonala et al., 2016; Puolakka et al., 2016); (B) examined the similarity of the network structure across age groups, because the role of and the interconnections between early risks may vary according to age; and (C) explored the extent to which symptoms with a more central role in the network (using specifically strength centrality representing the overall connectivity of the risks) are more strongly related to the risk of later health-related outcomes.

Methods

Study design and sample

The Cardiovascular Risk in Young Finns Study is an on-going follow-up study of the risk factors for coronary heart disease among Finnish children, adolescents and young adults (Raitakari et al., 2008). The first cross-sectional study was conducted in 1980, when age cohorts of 3-, 6-, 9-, 12-, 15- and 18-year-olds were randomly selected based on their social-security numbers, resulting in a total of 3,596 participants. Follow-up studies were conducted in 1983, 1986, 1989, 2001, 2007 and 2010/2011. The mean age in the younger group was 6.1 years and in 14.9 years in the older group.

The measurement of psychosocial risks (reported by the parents of the participants) was undertaken in 1980 . The health outcomes were collected from the last possible data-collection phases in 2007 and 2011 (depressive symptoms in 2007, 
body mass index and incident diabetes in 2010/2011, and intima media thickness in 2007). Information on all psychosocial risks in 1980 was available for 2,874 (1,289 men and 1,585 women) of the participants, who were thus included in the network analyses.

Written informed consent was obtained from participants who were at least nine years old, and from the parents of the younger ones. The research plan and datacollection procedures were accepted by the ethical review boards of five universities (Helsinki, Turku, Tampere, Kuopio and Oulu), and the data collection complied with WHO standards as well as the Helsinki Declaration.

Psychosocial factors in childhood

We assessed 4 childhood psychosocial factors (socioeconomic position, emotional climate, health behavioral environment, and stressful events) that have been proposed as central components of childhood psychosocial environment in previous theoretical models. The theoretical justification for choosing these components is based on the risky family model (Repetti et al., 2002; Taylor et al., 1997), which suggests that the most influential components in the family environment are the emotional climate on the one hand (how the parent interacts with the child) and socioeconomic factors (the family's material and educational resources) on the other. Of the many possible models, we chose this one, because it describes the range of normal family functioning - not solely pathological cases - and because it offers explanations on the biological pathways connecting early-life factors with health over the lifespan (e.g., related to changes in the endocrine and neurological functioning) (Taylor, 2010). Besides the risky family model, there is a convincing epidemiologic literature on childhood socioeconomic risks and health (Kawachi et al., 2010). Recently, the American Heart Association has published a statement on childhood psychosocial 
risks by pulling together evidence of the most influential risk factors (Suglia et al., 2017). Household emotional dysfunction, parental adverse health behaviors, and stressful events such as death or divorce are named as the most influential childhood risk factors (Suglia et al., 2017), which is in line with our conceptualization of childhood psychosocial risks factors.

As in previous studies (Pulkki-Råback et al., 2015; Elovainio et al., 2015b), the parents reported all the risk factors related to the psychosocial environment of their children (participants) in 1980, divided into the following domains: 1) child-specific stressful life events, 2) parental health behaviour, 3) parental socioeconomic status and 4) parental psychological problems (parental mental health problems, low parental satisfaction, low parental nurturance and heavy parental alcohol use). These factors were reported by the parents of the study participants via a paper-and-pencil questionnaire at the baseline examination in 1980, and were classified as binary risk factors using the following logic: $1=$ risk and $0=$ no risk (Evans et al., 2013) in accordance with previous studies (Pulkki-Råback et al., 2015) as follows:

\section{Child-specific stressful life-events}

Stressful life events were derived from scores for the following five experiences: moving house, change of school, divorce in the family, deaths of significant persons (parents, siblings) and hospitalization and/or serious illness in the family. Change of school was included as a stressful life event as it captures possible peer group change. Presence of each event during participant's childhood was assigned 1 point, otherwise the experience was assigned 0 .

\section{Parental health behaviour}

Parental health behaviors included the following 3 for both parent: mothers' and 
fathers' body mass index (BMI), smoking status, and levels of regular physical activity. The following cut-off points were used to establish the presence of high risk level (risk = 1) each category: mother and /or father obese $(\mathrm{BMI}>30 \mathrm{~kg} / \mathrm{m} 2)$; mother and / or father current smokers; mother and / or father reported no regular physical activity. Parental health behaviors were included as potential risk for two reasons. First, parental life style has been shown to predict the health behaviour of their offspring (Whitaker et al., 2010). Second, many of the health behavioural risks, such as obesity and sedentary life style, have been shown to be associated with cardiomatbolic outcomes and depressive symptoms in offsprings (Rosenstrom et al., 2013; McDermott et al., 1990).

\section{Parental socioeconomic status}

Four components were used to derive a risk score for the parental socioeconomic status: education $1=$ low (comprehensive school); $2=$ intermediate (secondary education): $3=$ high (academic, graduated from or studying at a polytechnic or university), occupational status (high non-manual, lower non-manual and manual), family income, and employment history (unemployment spells, long-term sickness absences spells, ill-health retirement). Family education and occupational status were defined according to the parent with the highest education or occupational status. The following cut-off points were used to define high risk levels (risk=1): low education, manual occupation, low income (lowest 25\%), and unsteady employment history (any of the following: unemployment spells, long-term sickness absences spells, parents on disability benefits). These cut-points have been used in previous Young Finns studies (Pulkki-Raback et al., 2015a).

\section{Parental psychological problems}

Parental psychological problems were derived based on four factors; mental health 
status, child-rearing style, life satisfaction, and alcohol abuse. Mental health status was measured with the following question: "Have you ever been diagnosed by a health professional as having a mental health problem? A "yes" response was considered as risk $(=1)$, "no" as no risk $(=0)$. Childrearing style was measured using a five-point scale derived from the Operation Family study (Katainen et al., 1999). This scale contains seven items assessing two different child-rearing dimensions, i.e. emotional significance (e.g., "I enjoy spending time with my child" (1=all the time...5=very seldom) and tolerance ("My child prevents me from fulfilling my needs" ( $1=$ all the time... $5=$ very seldom), which form a uniform scale (Cronbach's $\alpha$ $=.70$ ). Details of the scale have been reported elsewhere (Katainen et al., 1999). Providing a negative response, i.e. 4 or 5 on a 5 -point scale, to any item was used to indicate high risk (risk =1). The life satisfaction scale was also based on the Operation Family study (Katainen et al., 1999), and contains three questions that measure parental satisfaction as a mother/father, as a spouse, and with her/his work role (e.g. "I am satisfied as a mother"; $1=$ not at all, $5=$ very much) $(\alpha=.71)$. A response indicating dissatisfaction, i.e. 4 or 5 on a five-point scale, with any of the three life roles was classified as high risk (risk=1). Heavy alcohol use was included as a component of the psycho-emotional environment because of evidence indicating that unhealthy drinking is harmful to emotional development (Johnson and Leff, 1999). Parent's heavy alcohol use was measured as the number of heavy drinking occasions during year (heavy intoxication defined as $>6$ units) weekly with "Once a week" used as the cut-off point for high risk (risk=1).

Health outcomes in adulthood

Health outcomes measured in adulthood (27 - 32 years after the baseline, 
2007- 2010/2011) included depressive symptoms, body mass index (BMI), carotid artery wall thickness measured via intima media thickness (IMT), and incident type 2 diabetes. Depressive symptoms were assessed on a modified version of Beck's Depression Inventory (Elovainio et al., 2015a). The participants in the present study were asked to rate 21 items (e.g., "I often feel sad") on a five-point scale ranging from totally disagree (1) to totally agree (5). The Cronbach's alpha of the scale was 0.92 . BMI was calculated as weight $(\mathrm{kg}) /$ height $\left(\mathrm{m}^{2}\right)$ : the participants were weighed in light clothing without shoes on a set of digital scales with an accuracy of $0.1 \mathrm{~kg}$, whereas height was measured on a wall-mounted stadiometer accurate to $0.1 \mathrm{~cm}$, as described in detail previously (Raitakari et al., 2008). B-mode ultrasound studies of the left carotid artery were conducted in 2007 to measure IMT, with standardized protocols that have been reported previously (Raitakari et al., 2008). At least four measurements of the far wall were taken approximately $10 \mathrm{~mm}$ proximal to the bifurcation to derive maximum carotid IMT during both study years. The participants were classified as having type 2 diabetes if any of the following criteria were met: a) their fasting plasma glucose value was $\geq 7 \mathrm{mmol} / \mathrm{L}$ at any of the follow-up visits (2001, 2007, or 2010/2011); b) they reported having been given a type 2 diabetes diagnosis by a physician; c) their $\mathrm{HbA}_{1 \mathrm{c}}$ was $\geq 6.5 \%$ (48 $\left.\mathrm{mmol} / \mathrm{mol}\right)$ at the 2011 follow-up; d) they reported taking glucose-lowering medication at the 2007 or 2010/2011 follow-up; e) type 2 diabetes was diagnosed by a physician, based on the National Social Insurance Institution Drug Reimbursement Registry, which covers all Finnish citizens

Statistical analyses

The network structures of early psychosocial risks were estimated using the $\mathrm{R}$ -package IsingFit and Lasso (the least absolute shrinkage and selection operator) 
procedure for binary data (van Borkulo et al., 2014) first for all participants, and then separately for the three youngest and the three oldest age cohorts. This procedure is based on the Ising-model, which combines 11-regularized logistic regression with model selection based on the Extended Bayesian Information Criterion (EBIC). The differences in overall connectivity (defined as the weighted sum of the absolute connections (Barrat et al., 2004)) between the networks of younger and older age cohorts was assessed statistically using the Network Connection Test (NCT), which is implemented in the R-package of the NCT (Van Borkulo, 2015). The NCT is a twotailed permutation test that is a data-driven method in which all data are pooled and then randomly assigned to two groups, resulting in two estimated networks. We repeated this process 1500 times and that leads to a distribution of differences between networks given that the two groups come from the same population. It results in a distribution under the null hypothesis (on the assumption that both groups are equal), which can be used to test the significance of the difference between the original groups. The observed difference is considered significant at the threshold of .05. The EBIC is a function of the sample size and thus the lower the sample size, the more parsimonious the network structure would be. Our sample size was both relatively large and thus we had enough statistical power to detect possible differences between two groups.

To find out which of the psychosocial factors were the most central in the network (activating others or being activated by others), the position of each one was identified by computing the three local node-specific centrality measures: closeness, betweenness and node strength. (Barrat et al., 2004) Node strength measures the weighted number of connections of a focal node and thereby the degree to which it is involved in the network, and concerns its local structure. Closeness measures how 
close the focal node is to other nodes, and is inversely proportional to the mean shortest distance to all other nodes. Betweenness measures the degree to which the central node acts as a bridge that connects different parts of the network, and may reflect the degree to which the node can assert control over information flow through the network. Betweenness is measured as the number of times a node $\mathrm{X}$ lies on the shortest path between nodes $\mathrm{Y}$ and $\mathrm{Z}$.

To quantify the stability of the centrality indices we calculated the correlation stability coefficient (CS coefficient) using the R-package Bootnet (Epskamp, 2015) (with options nboots $=1500$ and $\mathrm{CaseN}=500$ ). The idea is to bootstrap various proportions of cases to assess the correlation between the original centrality indices and those obtained from subsets. It has been proposed that the CS coefficient should not be below 0.25 , and preferably above 0.5 , to interpret centrality differences (Epskamp et al., 2017). To assess the edge-weight accuracy, we estimated the 95\% confidence intervals of the edge weights using nonparametric bootstrapping as recommended by Epskamp and Fried (2017) and plotted (a) the edge-weight 95\% Cis (Supplement Figure s1), (b) the difference tests between all pairs of edge-weights (Supplement Figure s2) and (c) the difference test of the node strengths (Supplement Figure s3).

Multivariate linear and logistic regression analyses were conducted to assess whether the psychosocial factors that were central in the baseline network more strongly predicted health outcomes in adulthood. All the models were adjusted for age and gender because both age and gender have shown to be associated with all of the health outcomes included (Hankin and Abramson, 2001; Lahelma et al., 1999; MacIntyre and Hunt, 1997; McDonough et al., 1999; Mirowsky, 1996; Vahtera et al., 
2006; Sudlow et al., 2015). The STATA 13 statistical package was used for the final statistical analyses.

\section{Results}

Table 1 gives the characteristics of the study sample. The participants in the present study were more likely to be women $(\mathrm{p}<0.001)$, and to have a lower BMI $(\mathrm{p}=0.047)$ and a higher education in adulthood $(\mathrm{p}<0.001)$ than those who were lost-to-follow-up at some earlier point, but no other differences were detected.

Table 1 and Figure 1 about here

Figure 1 presents the results of the network analyses among all participants. All psychosocial risk domains seem to be relatively well connected, which could be interpreted, based on the visual perception of the network structure, to mean that health behaviours bridge socioeconomic problems and psychological problems. Life events have the fewest connections with factors in other domains. Figure 2 gives the individual centrality measures. The variables with the highest strength were those belonging to the socioeconomic-status risk domain, a low education, unemployment and a low income being the most heavily involved in the network. These factors also had the highest scores on betweenness and closeness. Risks related to specific life events such as divorce in the family or maternal hospitalization, however, were the least heavily involved.

Network stability in node betweenness $(C S=0.57)$, closeness $(C S=0.70)$ and strength $(\mathrm{CS}=0.52)$ were acceptable. The results of the nonparametric bootstrapping suggest that there were edge-weights differences especially between socioeconomic risks, but also between other risk domains (life-events) supporting the network structure presented in Figure 1. Similarly, the nodes with the largest strength (the 
socioeconomic risks, such as parental unemployment and education) also differed significantly from the most other nodes (Supplement Figures s1, s2, and s3).

Figure 2 about here

Although there were fewer connections between risks within the network among the older than among the younger participants (Figure 3), there were no differences in overall connectivity between the groups. The Network Connection Test confirmed that the difference in the strength of global connectivity was not statistically significant. The test statistic for the global-strength invariance test was $2.24(p=0.48)$. None of the tested edge-strength differences were statistically significant. There were no differences in the centrality indexes between the age groups (Supplement Figure s4).

Figure 3 about here

Table 2 presents the age- and gender-adjusted associations between psychosocial risks and health outcomes in adulthood. The more central risks predicted at least some of the outcomes relatively consistently. Thus, socioeconomic risks predicted depressive symptoms, elevated BMI and high IMT; parental obesity predicted elevated BMI and high IMT; and maternal physical activity predicted depressive symptoms. The less central risks related to life events did not predict any of the health outcomes in adulthood.

Table 2 about here

Discussion

In the current study, we applied the network approach to examine the network structure and inner connectivity of early psychosocial risk factors, focusing on whether more central and connected risk factors had stronger associations with health outcomes in adulthood. According to the results, risks related to socioeconomic status 
were the most strongly connected to each other and to other risks. The least strongly connected risks were those belonging to life-event domains such as parental hospitalization or divorce. There were no differences in overall connectivity between the three youngest and the three oldest participant groups. Nor were there any differences in local connectivity (the strength of the individual nodes was almost identical in the older and in the younger participants). Overall, our findings indicate that socioeconomic status and closely related risks may be more important in the accumulation of several risks, whereas specific risk from the life-events domain with small number of connections would have minimal influence in the total risk accumulation.

The risks that were central in the baseline network, such as socioeconomic risks were stronger predictors of higher BMI and IMT. Similarly, parental obesity predicted elevated BMI and high IMT and maternal physical activity predicted depressive symptoms of their children. Of the less central risks, only negative parental attitudes were associated with depressive symptoms in adulthood, and no other associations were found. These results indicate that the risk of developing health problems in adulthood depends on socioeconomic rather than other types of psychosocial risk factors. Similar findings, suggesting that especially socioeconomic risks predicted adulthood depression rather than risks from other domains, have been reported previously (Elovainio et al., 2015b), although not from a Network-analysis perspective. Because the same risks were also central in the psychosocial network they may be valuable targets in early-life risk prevention. The risks that are central in the network may, due to their position in the network, potentially explain rather large variation of other risks that in turn may also be associated with health outcomes. 
Affecting central risk may thus reduce negative health outcomes through multiple pathways.

Our finding of an association between a low socioeconomic status in childhood and poorer health in adulthood has been widely established in earlier research (Marmot et al., 1984; Marmot, 2004; Marmot, 2005; Elovainio et al., 2011), but the mechanisms linking socioeconomic exposures with future health remain unclear (Stringhini et al., 2017). The network approach offers a new way of testing the interrelations between multiple early-life socioeconomic risks. Such information may help in formulating hypothesis of the process linking these risks to later health outcomes. Longitudinal designs are needed, of course, to be able to test these hypotheses. More importantly, network models may help formulation of interventions, as they offer hypothesis about the potentially causal associations between individual risks. Thus, reducing a central symptom, in this case socioeconomic risks, may reduce (or prevent) the activity within the whole network. For example, a strategy that encourages people to obtain a high education not only improves employment prospects but also, and subsequently, may also have a positive effect on health behaviour. Our results further imply that focusing on preventing divorce, for instance, would not affect any other risks in the network and thus would be relatively inefficient as a method to reduce the negative outcomes examined in this study. One has to keep in mind, however, that our network analyses were based on cross-sectional data and thus longitudinal analyses are needed to determine the real causal associations. Although these preliminary results should be interpreted with caution, the network approach may facilitate more empirical, data-driven research resulting in more focused interventions based on the role of specific risks within a 
network. The model should be tested in future studies on a wider range of psychosocial risks than was possible in this study.

One the strengths of our study was that we included a large sample and used a prospective design with a long follow-up time to test the predicted outcomes. Although childhood factors could be measured more broadly, we were able to cover a relatively wide set of factors over several life-domains. The data attrition was considerable, but that does not seem to have led to significant bias in previous studies based on the same data set (Pulkki-Raback et al., 2015a; Elovainio et al., 2015b; Elovainio et al., 2016; Hakulinen et al., 2016). The limitations include the fact that the presented network was based on a between-subject design. Such networks may be representative of individuals if the groups are homogenous enough, and more research is warranted on whether the network structures presented here are indeed generalizable to individual participants. The robustness of our findings is supported by the fact that there were no statistically significant differences in overall connectivity between the three youngest and the three oldest age cohorts. The low prevalence of some of the risks may have caused the low connectivity, but not necessarily if the correlations were very high.

We were not able to use all the data in our network analyses due to missingness of the individual variables (risks). However, only about $20 \%$ of the participants had missing values and missing was not based on any one variable and thus it is not highly probable that the networks were biased because of missing values. Furthermore, prior studies using the same dataset showed that the associations of childhood psychosocial factors with cardiac and health behavior outcomes remained highly similar when computed in imputed data. These analyses suggested that there 
was no significant NMAR (not missing at random) in the data that would have affected the results. As the current study is conducted in the same dataset, using the same variables as, we feel rather confident to assume that imputation would not drastically change the results. (Pulkki-Råback et al., 2015)

To conclude, the network approach gives completely new insights into how childhood risks cluster together. Identifying dynamic networks of risks helps to better distinguish important risk factors from the less important ones. Such knowledge could facilitate the development of more sensitive and more targeted early prevention measures and interventions aimed at reducing the burden of risk related to several problems in the field of public health. Future studies could focus on the dynamic changes in risk networks over time, and on how these changes could predict health and other outcomes.

The authors have no financial relationships relevant to this article to disclose. The authors have no conflicts of interest to disclose. 
References

Baltrus PT, Everson-Rose SA, Lynch JW, et al. (2007) Socioeconomic position in childhood and adulthood and weight gain over 34 years: the Alameda County Study. Ann Epidemiol 17(8): 608-614.

Barrat A, Barthelemy M, Pastor-Satorras R, et al. (2004) The architecture of complex weighted networks. Proc Natl Acad Sci U S A 101(11): 37473752.

Borsboom D and Cramer AO. (2013) Network analysis: an integrative approach to the structure of psychopathology. Annu Rev Clin Psychol 9: 91-121.

Boschloo L, van Borkulo CD, Borsboom D, et al. (2016) A Prospective Study on How Symptoms in a Network Predict the Onset of Depression. Psychother Psychosom 85(3): 183-184.

Boschloo L, van Borkulo CD, Rhemtulla M, et al. (2015) The Network Structure of Symptoms of the Diagnostic and Statistical Manual of Mental Disorders. PLoS One 10(9): e0137621.

Bringmann LF, Vissers N, Wichers M, et al. (2013) A network approach to psychopathology: new insights into clinical longitudinal data. PLoS One 8(4): e60188.

Costantini G, Epskamp S, Borsboom D, et al. (2014) State of the art personality research: a tutorial on network analysis of personality data in R. J Res Pers 54: 13-29.

Cramer AO, Waldorp LJ, van der Maas HL, et al. (2010) Comorbidity: a network perspective. Behav Brain Sci 33(2-3): 137-150; discussion 150-193.

Dalege J, Borsboom D, van Harreveld F, et al. (2016) Toward a formalized account of attitudes: The Causal Attitude Network (CAN) model. Psychol Rev 123(1): 2-22.

DALYs GBD and Collaborators H. (2017) Global, regional, and national disabilityadjusted life-years (DALYs) for 333 diseases and injuries and healthy life expectancy (HALE) for 195 countries and territories, 1990-2016: a systematic analysis for the Global Burden of Disease Study 2016. Lancet 390(10100): 1260-1344.

DiPrete TA and Eirich GM. (2006) Cumulative advantage as a mechanism for inequality: A review of theoretical and empirical developments. Annual Review of Sociology 32: 271-297.

Elovainio M, Ferrie JE, Singh-Manoux A, et al. (2011) Socioeconomic differences in cardiometabolic factors: social causation or health-related selection? Evidence from the Whitehall II Cohort Study, 1991-2004. Am J Epidemiol 174(7): 779-789.

Elovainio M, Jokela M, Rosenstrom T, et al. (2015a) Temperament and depressive symptoms: what is the direction of the association? J Affect Disord 170: 203-212.

Elovainio M, Pulkki-Raback L, Hakulinen C, et al. (2015b) Childhood and adolescence risk factors and development of depressive symptoms: the 32-year prospective Young Finns follow-up study. J Epidemiol Community Health 69(11): 1109-1117. 
Elovainio M, Pulkki-Raback L, Hakulinen C, et al. (2016) Psychosocial environment in childhood and body mass index growth over 32years. Prev Med 97: 50-55.

Elovainio M, Pulkki-Raback L, Jokela M, et al. (2012) Socioeconomic status and the development of depressive symptoms from childhood to adulthood: a longitudinal analysis across 27 years of follow-up in the Young Finns study. Soc Sci Med 74(6): 923-929.

Epskamp S. (2015) bootnet: Bootstrap methods for various network estimation routines. $R$-package.

Epskamp S, Borsboom D and Fried EI. (2017) Estimating psychological networks and their accuracy: A tutorial paper. Behav Res Methods.

Epskamp S, Cramer AO, Waldorp LJ, et al. (2012) qgraph: network visualizations of relationships in psychometric data. . J Stat Softw 12: 1-18.

Evans GW, Dongping L and Whipple SS. (2013) Cumulative risk and child development. Psychological Bulletin 139: 1342-1396.

Frewen PA, Schmittmann VD, Bringmann LF, et al. (2013) Perceived causal relations between anxiety, posttraumatic stress and depression: extension to moderation, mediation, and network analysis. Eur J Psychotraumatol 4.

Fried EI, van Borkulo CD, Cramer A0, et al. (2017) Mental disorders as networks of problems: a review of recent insights. Soc Psychiatry Psychiatr Epidemiol 52(1): 1-10.

Hakulinen C, Pulkki-Raback L, Elovainio M, et al. (2016) Childhood Psychosocial Cumulative Risks and Carotid Intima-Media Thickness in Adulthood: The Cardiovascular Risk in Young Finns Study. Psychosom Med 78(2): 171181.

Hankin BL and Abramson LY. (2001) Development of gender differences in depression: an elaborated cognitive vulnerability-transactional stress theory. Psychol Bull 127(6): 773-796.

Johnson JL and Leff M. (1999) Children of substance abusers: Overview of research findings. Pediatrics 103: 1085-1099.

Juonala M, Pulkki-Raback L, Elovainio M, et al. (2016) Childhood Psychosocial Factors and Coronary Artery Calcification in Adulthood: The Cardiovascular Risk in Young Finns Study. JAMA Pediatr 170(5): 466-472.

Katainen S, Räikkönen K, Keskivaara P, et al. (1999) Maternal child-rearing attitudes and role satisfaction and children's temperament as antecedents of adolescent depressive tendencies: Follow-up study of 6- to 15-yearolds. Journal of Youth and Adolescence 28: 139-163.

Kawachi I, Adler NE and Dow WH. (2010) Money, schooling, and health: Mechanisms and causal evidence. Ann N Y Acad Sci 1186: 56-68.

Kendler KS. (2012) The dappled nature of causes of psychiatric illness: replacing the organic-functional/hardware-software dichotomy with empirically based pluralism. Mol Psychiatry 17(4): 377-388.

Kivimaki M, Smith GD, Juonala M, et al. (2006) Socioeconomic position in childhood and adult cardiovascular risk factors, vascular structure, and function: cardiovascular risk in young Finns study. Heart 92(4): 474-480.

Kossakowski JJ, Epskamp S, Kieffer JM, et al. (2016) The application of a network approach to Health-Related Quality of Life (HRQoL): introducing a new 
method for assessing HRQoL in healthy adults and cancer patients. Qual Life Res 25(4): 781-792.

Kruis J and Maris G. (2016) Three representations of the Ising model. Sci Rep 6: 34175.

Lahelma E, Martikainen P, Rahkonen O, et al. (1999) Gender differences in illhealth in Finland: patterns, magnitude and change. Soc Sci Med 48(1): 719.

MacIntyre S and Hunt K. (1997) Socio-Economic Position, Gender and Health: How Do They Interact?

Marmot GM. (2004) Status syndrome: How your social standing directly affects your health and life expectancy., London: Bloomsburry Publishing.

Marmot M. (2005) Social determinants of health inequalities. Lancet 365(9464): 1099-1104.

Marmot MG and A F. (1996) Socioeconomic factors in CHD prevention. In: OrthGomér K and Schneiderman N (eds) Behavioral medicine approaches to cardiovascular disease prevention. New Jersey: Lawrence Erlbaum, 21-41.

Marmot MG, Shipley MJ and Rose G. (1984) Inequalities in death--specific explanations of a general pattern? Lancet 1(8384): 1003-1006.

Marmot MG, Smith GD, Stansfeld S, et al. (1991) Health inequalities among British civil servants: the Whitehall II study. Lancet 337(8754): 13871393.

McDermott RJ, Hawkins WE, Marty PJ, et al. (1990) Health behavior correlates of depression in a sample of high school students. J Sch Health 60(8): 414417.

McDonough P, Williams DR, House JS, et al. (1999) Gender and the socioeconomic gradient in mortality. J Health Soc Behav 40(1): 17-31.

McNally RJ, Mair P, Mugno BL, et al. (2017) Co-morbid obsessive-compulsive disorder and depression: a Bayesian network approach. Psychol Med 47(7): 1204-1214.

Mirowsky J. (1996) Age and the gender gap in depression. J Health Soc Behav 37(4): 362-380.

Okasha M, McCarron P, McEwen J, et al. (2003) Childhood social class and adulthood obesity: findings from the Glasgow Alumni Cohort. J Epidemiol Community Health 57(7): 508-509.

Pulkki-Råback L, Elovainio M, Hakulinen C, et al. (2015) Cumulative Effect of Psychosocial Factors in Youth on Ideal Cardiovascular Health in Adulthood. The Cardiovascular Risk in Young Finns Study. . Circulation.

Pulkki-Raback L, Elovainio M, Hakulinen C, et al. (2015a) Cumulative effect of psychosocial factors in youth on ideal cardiovascular health in adulthood: the cardiovascular risk in young Finns study. Circulation 131(3): 245-253.

Pulkki-Raback L, Elovainio M, Hakulinen C, et al. (2015b) Cumulative effect of psychosocial factors in youth on ideal cardiovascular health in adulthood: the Cardiovascular Risk in Young Finns Study. Circulation 131(3): 245253.

Puolakka E, Pahkala K, Laitinen TT, et al. (2016) Childhood Socioeconomic Status in Predicting Metabolic Syndrome and Glucose Abnormalities in Adulthood: The Cardiovascular Risk in Young Finns Study. Diabetes Care 39(12): 2311-2317. 
Raitakari OT, Juonala M, Rönnemaa T, et al. (2008) Cohort profile: The Cardiovascular risk in young Finns study. International Journal of Epidemiology: 1-7.

Repetti RL, Taylor SE and Seeman TE. (2002) Risky families: family social environments and the mental and physical health of offspring. Psychological Bulletin 128(2): 330-366.

Rosenstrom T, Jokela M, Hintsanen M, et al. (2013) Body-image dissatisfaction is strongly associated with chronic dysphoria. J Affect Disord 150(2): 253260.

Singh-Manoux A, Adler NE and Marmot MG. (2003) Subjective social status: its determinants and its association with measures of ill-health in the Whitehall II study. Social Science \& Medicine 56(6): 1321-1333.

Stringhini S, Carmeli C, Jokela M, et al. (2017) Socioeconomic status and the $25 \mathrm{x}$ 25 risk factors as determinants of premature mortality: a multicohort study and meta-analysis of 1.7 million men and women. Lancet.

Sudlow C, Gallacher J, Allen N, et al. (2015) UK biobank: an open access resource for identifying the causes of a wide range of complex diseases of middle and old age. PLoS Med 12(3): e1001779.

Suglia SF, Koenen KC, Boynton-Jarrett R, et al. (2017) Childhood and Adolescent Adversity and Cardiometabolic Outcomes: A Scientific Statement From the American Heart Association. Circulation.

Tamayo T, Christian H and Rathmann W. (2010) Impact of early psychosocial factors (childhood socioeconomic factors and adversities) on future risk of type 2 diabetes, metabolic disturbances and obesity: a systematic review. BMC Public Health 10: 525.

Taylor SE. (2010) Mechanisms linking early life stress to adult health outcomes. Proc Natl Acad Sci U S A 107(19): 8507-8512.

Taylor SE, Repetti RL and Seeman T. (1997) Health psychology: What is an unhealthy environment and how does it get under the skin? Annual Review of Psychology 48: 411-447.

Vahtera J, Kivimaki M, Vaananen A, et al. (2006) Sex differences in health effects of family death or illness: are women more vulnerable than men? Psychosomatic Medicine 68(2): 283-291.

Van Borkulo C. (2015) Network Comparison Test:Permutation-Based Test of Differences in Strength of Networks. https://github.com/cvborkulo/NetworkComparisonTest.

van Borkulo C, Borsboom D, Epskamp S, et al. (2014) A new method for constructing networks from binary data. Sci Rep 4: 5918.

van Borkulo C, Boschloo L, Borsboom D, et al. (2015) Association of Symptom Network Structure With the Course of [corrected] Depression. JAMA Psychiatry 72(12): 1219-1226.

Whitaker KL, Jarvis MJ, Beeken RJ, et al. (2010) Comparing maternal and paternal intergenerational transmission of obesity risk in a large population-based sample. Am J Clin Nutr 91(6): 1560-1567. 


\begin{tabular}{|c|c|c|}
\hline & Mean or $\%$ & SD or $n$ \\
\hline Age in 1980 & 9.8 & 4.9 \\
\hline Male sex $(\% / \mathrm{n})$ & 49 & 1289 \\
\hline \multicolumn{3}{|l|}{ Childhood risks (yes) } \\
\hline Maternal obesity & 7.9 & 272 \\
\hline Paternal obesity & 8.6 & 267 \\
\hline Maternal smoking & 16.7 & 579 \\
\hline Paternal smoking & 32.1 & 1116 \\
\hline Maternal physical activity & 29.9 & 1058 \\
\hline Paternal physical activity & 20.41 & 712 \\
\hline Moving residence & 5.1 & 179 \\
\hline Change of school & 14.9 & 527 \\
\hline Divorce & 14.8 & 531 \\
\hline Mother hospitalized & 2.7 & 93 \\
\hline Father hospitalized & 2.2 & 66 \\
\hline Mental-health problems & 2.6 & 91 \\
\hline Low education & 34.7 & 1228 \\
\hline Low occupational status & 39.6 & 1387 \\
\hline Low income & 27.5 & 950 \\
\hline Unemployment & 10.3 & 370 \\
\hline Heavy alcohol use & 13.2 & 468 \\
\hline Negative parental attitudes & 18.2 & 656 \\
\hline Dissatisfaction & 7.5 & 268 \\
\hline \multicolumn{3}{|l|}{ Outcomes in adulthood } \\
\hline Depressive symptoms 2007 (range 1 - 5) & 2.06 & 0.67 \\
\hline Body-mass index $(\mathrm{kg} / \mathrm{m} 2)$ in $2010 / 2011$ & 26.5 & 5.10 \\
\hline Intima Media thickness 2007 (mm) & 0.63 & 0.10 \\
\hline Type 2 Diabetes in 2010/2011 & 3.34 & 120 \\
\hline
\end{tabular}


Table 2. The centrality (strength) of the psychosocial risks and their associations with health-related outcomes

\begin{tabular}{llllll}
\hline & $\begin{array}{l}\text { Centrality of } \\
\text { factor in the } \\
\text { network }\end{array}$ & $\begin{array}{l}{ }^{1} \text { Depressive } \\
\text { symptoms }\end{array}$ & ${ }^{1}$ BMI & ${ }^{1}$ IMT & ${ }^{2}$ Type 2 Diabetes \\
\cline { 2 - 6 } & Strength & B / SE & B / SE & B / SE & OR $(95 \%)$ \\
\cline { 2 - 6 } Maternal obesity & 2.58 & $0.09 / 0.03$ & $\mathbf{3 . 5 6} / \mathbf{0 4 2}$ & $\mathbf{0 . 1 8} / \mathbf{0 . 0 1}$ & $\mathbf{3 . 4 8}(\mathbf{2 . 2 2 , 5 . 4 6 )}$ \\
Paternal obesity & 1.07 & $0.00 / 0.05$ & $\mathbf{2 . 5 3} / \mathbf{0 . 4 1}$ & $0.01 / 0.01$ & $1.38(0.74,2.57)$ \\
Maternal smoking & 2.22 & $0.00 / 0.04$ & $0.33 / 0.31$ & $0.01 / 0.01$ & $1.38(0.86,2.21)$ \\
Paternal smoking & 2.78 & $0.03 / 0.03$ & $0.41 / 0.24$ & $0.01 / 0.00$ & $\mathbf{1 . 5 6}(\mathbf{1 . 0 6 , 2 . 3 0})$ \\
Maternal physical activity & 3.24 & $\mathbf{0 . 0 6} / \mathbf{0 . 0 3}$ & $0.00 / 0.24$ & $0.01 / 0.00$ & $1.46(0.98,2.13)$ \\
Paternal physical activity & 3.09 & $0.02 / 0.04$ & $0.14 / 0.27$ & $0.00 / 0.00$ & $1.02(0.65,1.62)$ \\
Moving residence & 2.50 & $0.06 / 0.06$ & $0.67 / 0.51$ & $0.02 / 0.01$ & $1.24(0.61,2.51)$ \\
School change & 1.98 & $0.02 / 0.04$ & $0.56 / 0.31$ & $0.00 / 0.01$ & $1.39(0.89,2.16)$ \\
Divorce & 0.00 & $0.06 / 0.04$ & $0.31 / 0.33$ & $0.00 / 0.01$ & $0.97(0.59,1.58)$ \\
Mother hospital & 0.00 & $-0.09 / 0.09$ & $0.99 / 0.64$ & $0.01 / 0.01$ & $\mathbf{2 . 7 2}(\mathbf{1 . 2 7}, \mathbf{5 . 8 1})$ \\
Father hospital & 1.85 & $0.14 / 0.11$ & $-0.27 / 0.81$ & $0.01 / 0.01$ & $1.93(0.75,4.97)$ \\
Depressive symptoms & 1.43 & $0.17 / 0.10$ & $0.18 / 0.81$ & $0.01 / 0.01$ & $1.59(0.63,4.01)$ \\
Low education & 4.70 & $0.03 / 0.03$ & $0.41 / 0.24$ & $\mathbf{0 . 0 1} / \mathbf{0 . 0 0}$ & $\mathbf{1 . 4 7}(\mathbf{1 . 0 1}, \mathbf{2 . 1 4})$ \\
Low occupational status & 2.95 & $0.03 / 0.03$ & $\mathbf{0 . 6 1} / \mathbf{0 . 2 2}$ & $\mathbf{0 . 0 1} / \mathbf{0 . 0 0}$ & $1.13(0.77,1.65)$ \\
Low income & 3.59 & $\mathbf{0 . 1 1} / \mathbf{0 . 0 3}$ & $\mathbf{0 . 7 8} / \mathbf{0 . 2 5}$ & $\mathbf{0 . 0 1} / \mathbf{0 . 0 0}$ & $\mathbf{1 . 6 5}(\mathbf{1 . 1 2 , 2 . 4 2})$ \\
Unemployment & 5.14 & $0.07 / 0.05$ & $\mathbf{0 . 8 4} / \mathbf{0 . 3 7}$ & $0.01 / 0.00$ & $\mathbf{1 . 7 1}(\mathbf{1 . 0 7 , 2 . 7 7})$ \\
Heavy alcohol use & 2.49 & $0.09 / 0.04$ & $0.08 / 0.32$ & $0.00 / 0.01$ & $0.93(0.52,1.64)$ \\
Negative parental attitudes & 0.90 & $\mathbf{0 . 1 5} / \mathbf{0 . 0 4}$ & $0.48 / 0.29$ & $0.00 / 0.01$ & $0.88(0.53,1.46)$ \\
Dissatisfaction & 1.27 & $0.11 / 0.21$ & $-0.27 / 0.40$ & $-0.01 / 0.01$ & $0.69(0.30,1.59)$ \\
\hline
\end{tabular}

Significant associations in bold, ${ }^{1}=$ linear regression, ${ }^{2}=$ logistic regression 
Figure captions:

Figure 1. Network (Lasso regularized Ising - models) of early psychosocial risks ( $\mathrm{N}=$ 2580). The nodes are individual psychosocial risks and edges the independent (adjusted for the effects of all the other nodes) association between them (the green edges are positive and red negative associations).

Figure 2. Centrality measures of individual psychosocial risks. The measures are: (1) node strength, quantifying how well (strongly) a node is indirectly or directly connected to other nodes; (2) closeness, quantifying how well a node is indirectly connected to other nodes; and (3) betweenness, quantifying how important a node is in the average path between two other nodes.

Figure 3. Network (Lasso regularized Ising - models) of early psychosocial risks in younger and older cohorts. The nodes are individual psychosocial risks and the edges are independent (adjusted for the effects of all the other nodes) associations between them (the green edges are positive and the red are negative associations). 

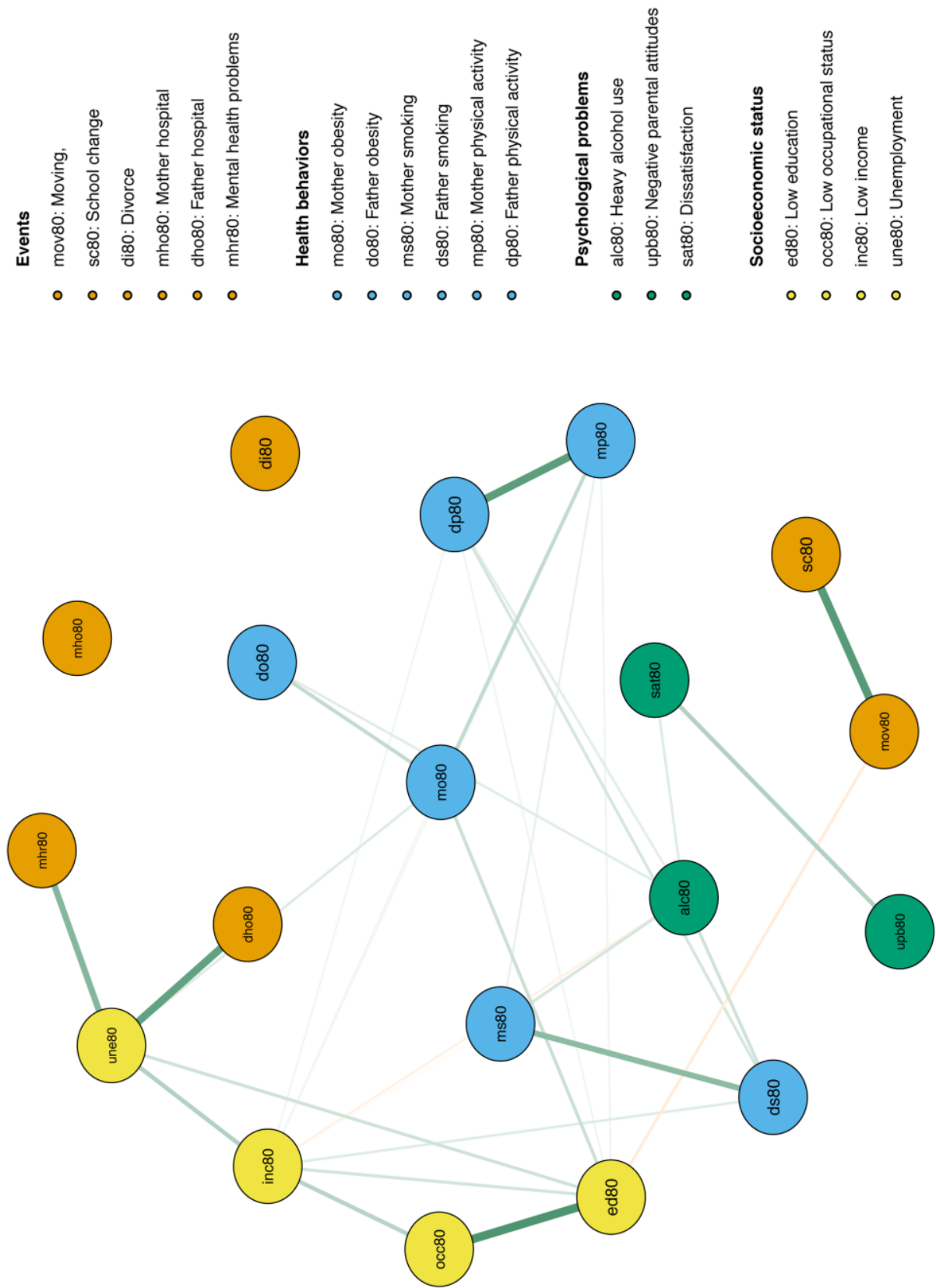

Figure 1 

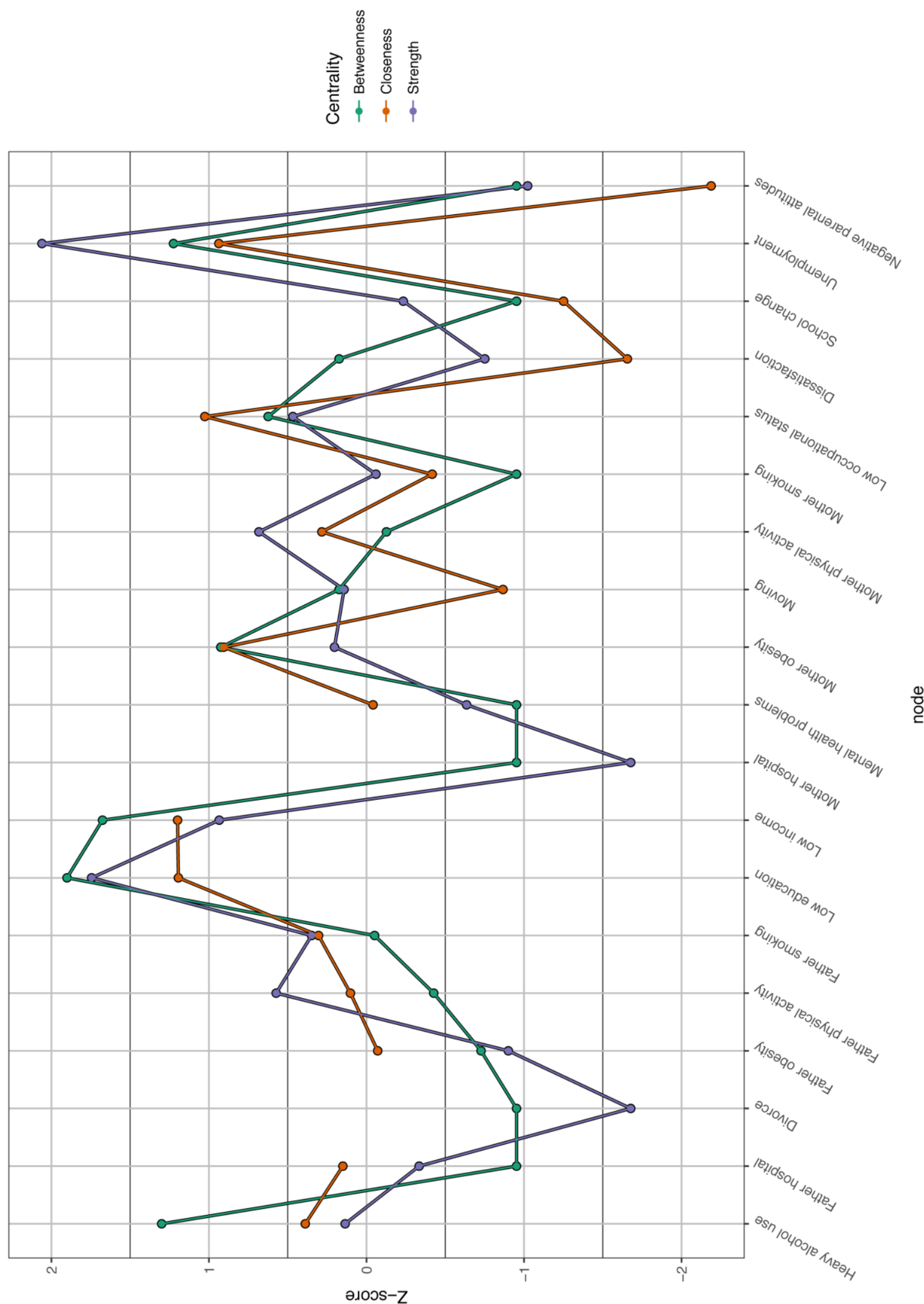

Figure 2. 


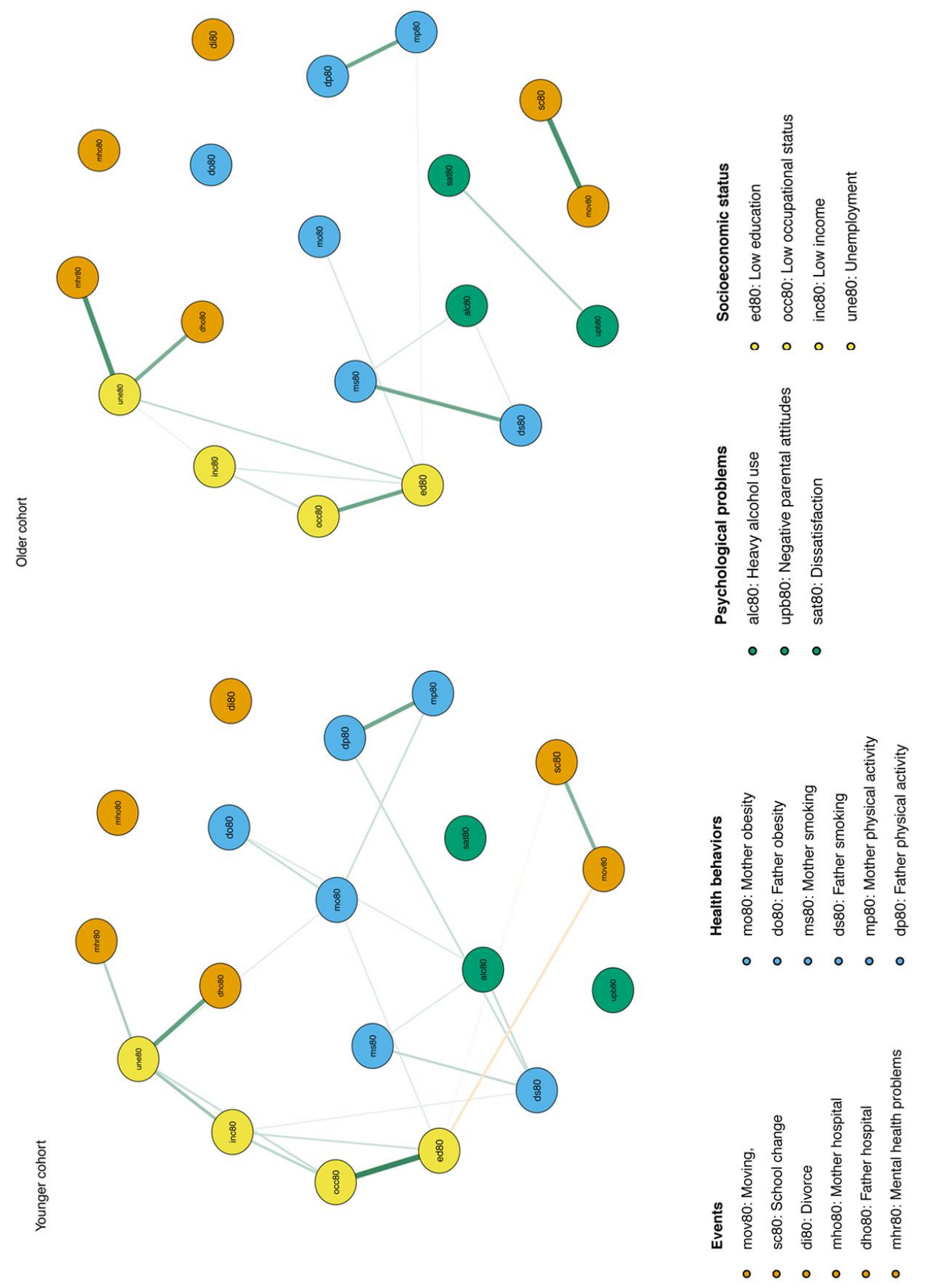

Figure 3. 